\title{
MATRIX INEQUALITIES BY MEANS OF BLOCK MATRICES
}

\author{
FUZHEN ZHANG
}

\begin{abstract}
We first show a weak log-majorization inequality of singular values for partitioned positive semidefinite matrices which will imply some existing results of a number of authors, then present some basic matrix inequalities and apply them to obtain a number of matrix inequalities involving sum, ordinary product and Hadamard product.
\end{abstract}

Mathematics subject classification (2000): 15A45.

Key words and phrases: Eigenvalue inequality; Hadamard (Schur) product; matrix inequality; positive semidefinite matrix; singular value inequality; unitarily invariant norm; weak log-majorization.

\section{REFERENCES}

[1] A. ALBERT, Conditions for positive and nonnegative definiteness in terms of pseudoinverses, SIAM J. Appl. Math, Vol. 17, No. 2, March 1969.

[2] P. A. BEKKER, The positive semidefiniteness of partitioned matrices, Linear Algebra and Its Applications 111:261-278 (1988).

[3] R. Bhatia, Matrix Analysis, Springer-Verlag, New York, 1997.

[4] R. Bhatia And F. Kittaneh, Norm Inequalities for Positive Operators, Letters in Math Phys. 43: 225-231 (1998).

[5] C. H. FitzGerald AND R. A. HoRn, On fractional Hadamard powers of positive definite matrices, J. of Math Analysis and Appl. 61, pp. 633-642 (1977).

[6] P. R. Halmos, Linear Algebra Problem Book, Mathematical Association of America, Washington, DC, 1995.

[7] R. A. Horn AND C. R. Johnson, Topics in Matrix Analysis, Cambridge University Press, New York, 1991.

[8] C. R. Johnson, Partitioned and Hadamard product matrix inequalities, J. Res. Nat. Bur. Standards, 83: 585-591 (1978).

[9] V. V.PRASOLOV, Problems and Theorems in Linear Algebra, American Mathematical Society, Providence, RI, 1994.

[10] G. VISICK, A quantitative version of the observation that the Hadamard product is a principal submatrix of the Kronecker product, Linear Algebra and Its Applications 304 (2000) 45-68.

[11] G. VISICK, An algebra relationship between the Hadamard and Kronecker product with some applications, Bull. Soc. Math. Belg. 42(1990), 3, Ser. B, pp. 275-283.

[12] B.-Y. WANG, B.-Y. XI AND F. ZHANG, Some inequalities for sum and product of positive semidefinite matrices, Linear Algebra and Its Applications, 293 (1999) 39-49.

[13] X.-Z.ZHAN, Singular values of differences of positive semidefinite matrices, SIAM Matrix Analysis and Appl. Vol. 22, No. 3 (2000), 819-823.

[14] F. ZHANG, Another Proof of a Singular Value Inequality Concerning Hadamard Products of Matrices, Linear and Multilinear Algebra, Vol. 22 (1988) 307-311.

[15] F. ZHANG, Notes on Hadamard products of matrices, Linear and Multilinear Algebra, Vol. 25 (1989) 237-242.

[16] F. ZHANG, Schur complements and matrix inequalities in the Löwner ordering, Linear Algebra and Its Applications 321 (2000) 399-410.

[17] F. ZHANG, Matrix Theory: Basic Results and Techniques, Springer-Verlag, New York, 1999. 